Ann. Abeille, Ig62, 5 (3), 233-246

\title{
ESSAIS PRÉLIMINAIRES DE MESURE DE LA CAPACITÉ D'ÉLEVAGE DANS LA RUCHE
}

\author{
II. MONTMGNER \\ Laboratoire de Psychophysiologie, Faculti des Sitences, Nancy; \\ Station de Recherches sur l'Abeille et les Insectes sociaux, Bures-sur-Yzlette (Scine-et-Oise)
}

SOMMAIRE

L'auteur étudie les principales influences qui s'esercent sur l'acceptation des crufs et l'élevage des larves dans la colonie d'abeilles. Deux cas sont considérés : celui de la ruche normale ct celui de la ruche en état de disette. On constate que la présence de couvain opereulé et la distance des larves a la reine sont deux facteurs déterminants.

\section{INTRODUCTION}

L'acceptation des cufs et l'élevage des larves constituent des facteurs qui influencent de façon essentielle le bon développement de la colonie d'abeilles. I)ifférents auteurs ont remarqué que ces aspects du comportement social ne sont pas simples et ont essayé d'analyser les influences qui s'exercent sur la capacité d'élevage des colonies.

Ainsi, il s'est avéré que le taux d'élevage n'est pas rigoureusement proportionnel à l'importance de la population. TARANOv (1946) a montré que l'élevage le plus intense par Abeille est réalisé dans des colonies de I $\mathrm{kg}(3,85$ larves par Abeille adulte) et qu'il décroît lorsque ce poids est dépassé. Ces résultats ont été confirmés par une élève de Musalewsky, Denissova (I956).

Il est possible qu'il en soit ainsi, mais il reste encore à préciser ce qu'est le rendement d'élevage d'une ruche. En effet, cet élevage dépend de nombreux facteurs qui interfèrent souvent.

Chauvin (I956) a établi que l'élevage du couvain est soumis à de nombreuses fluctuations; il a pu calculer expérimentalement un rendement de transformation cufs-larves de l'ordre de $90 \mathrm{p}$. roo et larves-nymphes pouvant varier de $72 \mathrm{p}$. Ioo à 80 p. IoO.

Il n'est pas rare, en effet, de constater dans une ruche des destructions d'œufs qui peuvent atteindre de grandes proportions, comme nous le verrons plus loin. Nous nous sommes donc proposé d'étudier systématiquement les facteurs responsables de ces déficiences d'élevage. 


\section{MATÉRIEL, ETT TECHNIQUE}

Nos premières observations sur l'élimination spontanée des larves ont été faites dans des ruches qui servaient à l'étude expérimentale de la ponte de la reine. Nous avons toujours conservé dans ces ruches la même disposition des cadres afin de maintenir des conditions d'élevage identiques.

Les expériences ont ensuite porté sur des ruches normales à Io cadres que nous avons divisées en deux parties par une grille à reine verticale, la zone où se trouvait la reine comportant 3 cadres. Les cadres étant numérotés de I à ı, comme le schéma l'indique (fig. I), cerix qui sont placés en I et 3 sont choisis converts d'wufs et de couvain. Le cadre 2 est introduit vide et remplacé dès que la ponte de la reine est devenue trop importante pour qu'elle puisse se poursuivre normalement.

Ainsi, la ponte n'est pas perturbée et la vie de la colonie se poursuit normalement grâce à l'apport sensiblement constant des abeilles adultes qui naissent en i et 3 .

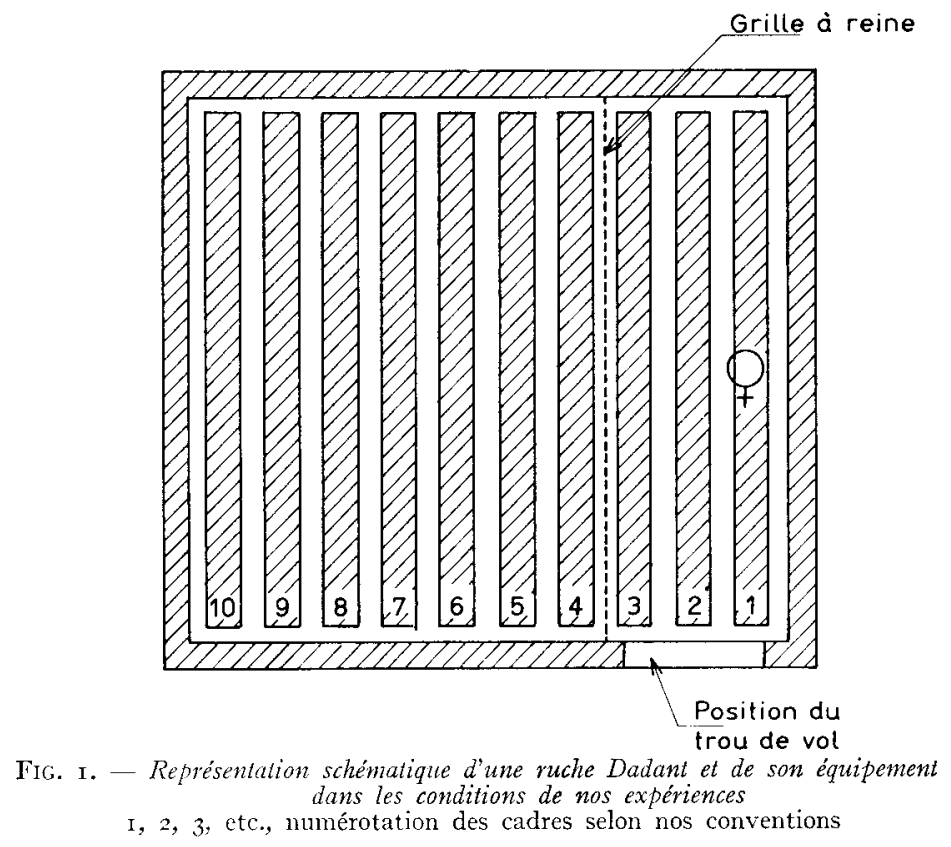

OBSERVATIONS ET RÉSULTATS

\section{A. - INFLUENCE DE LA TEMPÉRATURE INTERNE DE LA RUCHE}

Il n'est pas douteux que l'élevage du couvain est soumis à la régulation thermique dont la ruche est le siège ; c'est ainsi que les oufs et les larves figurant à la périphérie d'un cadre ont parfois peu de chances de donner des adultes. Mais la régulation de 1a température étant elle-même fonction du nombre d'abeilles, il en résulte que ce dernier facteur conditionne finalement l'élevage des larves à la périphérie des cadres (Notons que c'est là le seul avantage d'avoir une forte colonie, puisque TARANov, rappelons-le, a montré que l'élevage des larves était maximum pour les colonies de $I$ kg.) 


\section{B. - DESTRUCTION D'GUFS DANS LA PARTIE ORPHELINE DE LA RCCHE COMPORTANT PEU DE RÉSERVES ET PEU DF, COUVAIN}

Ayant été amenés à constater, dans la ruche en état de disette, des destructions d'œufs et de larves importantes, nous avons placé les cadres venant de 2 dans la partie orpheline, à mesure qu'ils étaient couverts d'œufs.

a) Dans une première série d'expériences, nous avons mis les cadres 2 en position 9 dans la partie orpheline (voir le schéma de la répartition des cadres dans la ruche).

Au bout de deux ou trois jours, selon le nombre d'œufs présents au départ, tout était nettoyé sur le cadre 9 , à l'exception de quelques œufs dispersés.

Cette expérience, répétée plusieurs fois sur différentes ruches, nous a toujours montré que la destruction des œufs s'y faisait d'une façon presque linéaire.

La figure 2 donne pour 6 ruches les courbes de destructions d'œufs sur les cadres placés en position 9, en période de disette. En abscisses, la date à laquelle s'est faite la mesure du nombre d'œufs présents sur le cadre 9 , et en ordonnées, la quantité d'œufs restants.

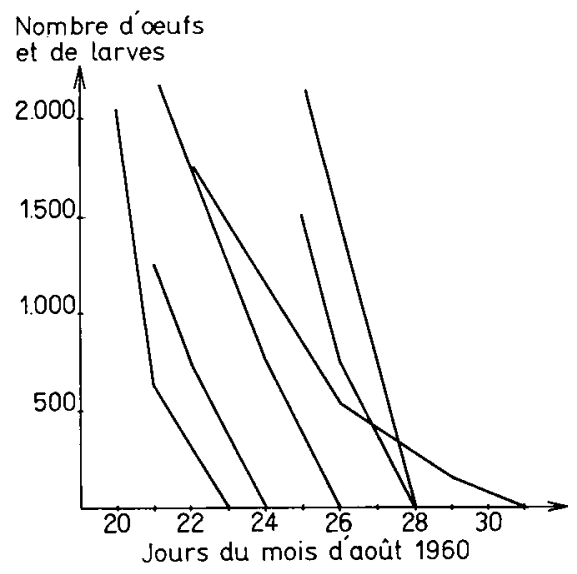

FiG. 2. - Évolution de l'élevage des larves sur le cadre g jusqu'à operculation dans des ruches en état de disette

b) Dans une deuxième série d'expériences, nous avons mis les cadres 2 pleins d'œufs en position 6,7 et 8 .

De la même façon que précédemment, nous avons établi les courbes de pourcentage d'œufs, puis de larves acceptées jusqu'à leur operculation, en fonction du temps, et ceci dans 4 ruches (fig. 3 ).

Le début des paliers horizontaux correspond à l'operculation du couvain, ou parfois à des larves âgées qui seront finalement acceptées.

Signalons encore qu'il arrive que l'acceptation soit effective en 8 dans les ruches très actives mais que, même dans ce cas, elle est troujours très faible.

c) Dans une troisième série d'expériences. - Le cadre 2 étant placé en position 4 ou 5 , nous avons obtenu les résultats qui sont représentés graphiquement sur la figure 4 . 
Ces courbes nous montrent de façon indubitable que, jusqu'à l'operculation du couvain, des oufs et des larves sont continuellement détruits ou mangés.

En outre, cette destruction ne se fait pas au hasard, mais elle semble fonction de la distance des œufs et des larves à la reine. Cette dernière représenterait donc un

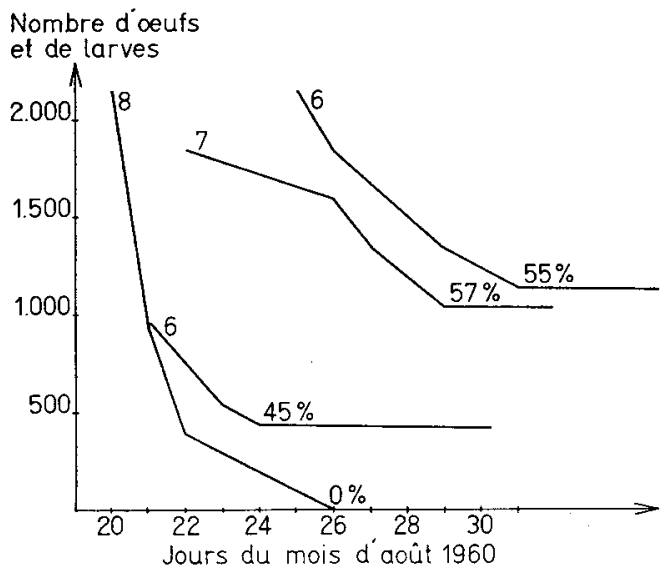

FIG. 3. - Évolution de l'élevage des larves sur les cadres 6,7 et 8 jusqu'à operculation dans des ruches en état de disette

Le pourcentage indiqué est celui des larves définitivement acceptées et operculées

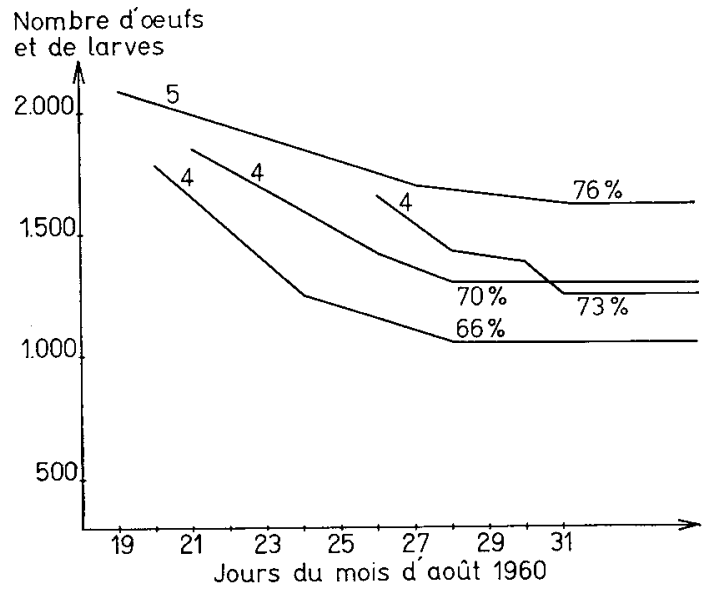

FIG. 4. - Évolution de l'élevage des larves sur les cadres 4 et 5 jusqu'à operculation dans des ruches en état de disette

Le pourcentage indiqué est celui des larves définitivement acceptées et operculées

point central dont la zone d'influence conditionnerait l'adoption des œufs et l'élevage des larves.

Nous avons alors essayé de préciser ces résultats dans les conditions de la ruche normale. 


\section{C. - AIOPTION DES GEUFS ET ÉLEVAGE DES LARVES DANS LES CONDITIONS NORMALES DE LA RUCHE SAINE,}

Précisons d'abord que par "ruche saine ", nous désignons une population assez forte avec des réserves et du couvain operculé en quantité telle que l'activité première de la ruche puisse se conserver pendant longtemps après le début de l'expérience de séparation d'un compartiment convenant à la reine.

\section{Influence de la distance des cuts et larves à la reine}

Ayant été amenés à envisager ce facteur pour l'étude de la ruche en état de disette, nous venons de montrer que les oufs et larves sont d'autant mieux acceptés qu'ils se trouvent plus près de la reine.

Nous avons renouvelé nos expériences en utilisant le même dispositif expérimental ; cette fois, les cadres fraîchement garnis d'œufs sont introduits dans la partie orpheline dans des conditions différentes de celles qui règnent dans la ruche en état de disette.

De cette façon, nous avons pu étudier l'influence de la proximité du couvain operculé et des réserves, ainsi que de leur abondance, sur l'élevage de la colonie et du nombre de larves à nourrir.

La reine se trouvant isolée sur les trois premiers cadres, 4 ruches furent mises en expérience à partir du 20 juillet Ig6r. Elles comprenaient toutes des populations d'abeilles de race italienne, sensiblement égales (ruches contenant environ $2,5 \mathrm{~kg}$ d'abeilles). En outre, au départ, elle possédaient toutes sensiblement la même quantité de couvain operculé et de réserves (miel et pollen). Dans une $5^{\mathrm{e}}$ ruche, l'élevage des larves a été continuellement suivi pendant un mois et demi.

Ces expériences nous ont donné les résultats consignés dans les tableaux I et 2 et que l'on peut résumer comme suit:

TABLEAU I

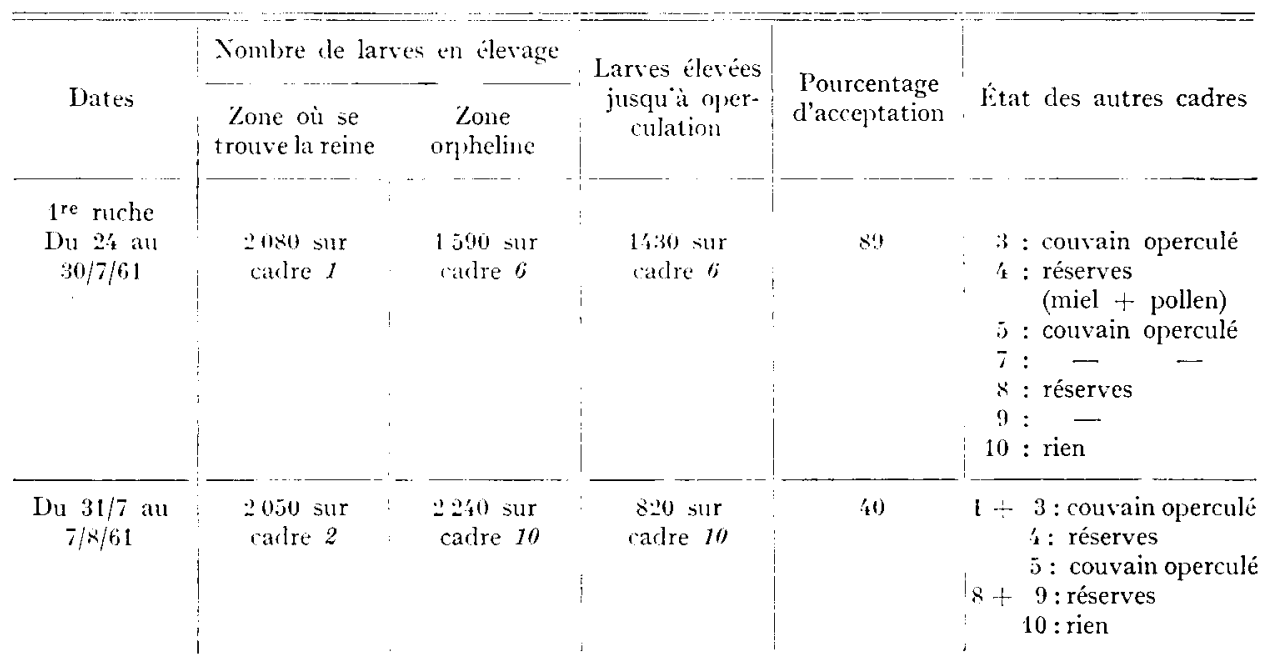

Annales de l'Abeille. - I962. 
TABLEAU I (Suite)

\begin{tabular}{|c|c|c|c|c|c|}
\hline \multirow{2}{*}{ Dates } & \multicolumn{2}{|c|}{ Nombre de larves en élevage } & \multirow{2}{*}{$\begin{array}{c}\text { Larves élevées } \\
\text { jusqu'à oper- } \\
\text { culation }\end{array}$} & \multirow{2}{*}{$\begin{array}{l}\text { Pourcentage } \\
\text { d'acceptation }\end{array}$} & \multirow{2}{*}{ État des autres cadres } \\
\hline & $\begin{array}{c}\text { Zone où se } \\
\text { trouve la reine }\end{array}$ & $\begin{array}{l}\text { Zone } \\
\text { orpheline }\end{array}$ & & & \\
\hline $\begin{array}{c}2^{2} \text { ruche } \\
\text { Du } 24 / 7 \text { au } \\
28 / 7 / 61\end{array}$ & $\begin{array}{l}620 \text { sur } \\
\text { cadre } 1 \\
830 \text { sur } \\
\text { cadre } 3\end{array}$ & $\begin{array}{l}1100 \text { sur } \\
\text { cadre } 6 \\
2200 \text { sur } \\
\text { cadre } 9\end{array}$ & $\begin{array}{l}690 \text { sur } \\
\text { cadre } 6 \\
1100 \text { sur } \\
\text { cadre } 9\end{array}$ & $\begin{array}{l}62 \\
50\end{array}$ & $\begin{aligned} & 4: \text { réserves } \\
& 5+\quad 7: \text { couvain operculé } \\
& 8+9: \text { réserves } \\
& 10: \text { rien }\end{aligned}$ \\
\hline $\begin{array}{c}3^{e} \text { ruche } \\
\text { Du } 26 / 7 \text { au } \\
1 / 8 / 61\end{array}$ & $\begin{array}{l}660 \text { sur } \\
\text { cadre } 3\end{array}$ & $\begin{array}{l}1306 \text { sur } \\
\text { cadre } 9\end{array}$ & $\begin{array}{l}900 \text { sur } \\
\text { cadre } 9\end{array}$ & 65 & $\begin{array}{l}1+3: \text { couvain operculé } \\
4+5:- \text { - } \\
6+7: \text { réserves en gde } \\
\quad \text { quantité } \\
8+10: \text { couvain opercule }\end{array}$ \\
\hline $\begin{array}{c}4^{\ominus} \text { ruche } \\
\text { Du } 20 \text { au } \\
26 / 7 / 61\end{array}$ & $\begin{array}{l}1920 \text { sur } \\
\text { cadre } 2 \\
360 \text { sur } \\
\text { cadre } 3\end{array}$ & $\begin{array}{l}1520 \text { sur } \\
\text { cadre } 6 \\
2150 \text { sur } \\
\text { cadre } 9\end{array}$ & $\begin{array}{l}1180 \text { sur } \\
\text { cadre } 6 \\
400 \text { sur } \\
\text { cadre } 9\end{array}$ & 20 & $\begin{aligned} 1+3 & : \text { couvain operculé } \\
4 & :- \\
& + \text { réserves } \\
5 & : \text { couvain operculé } \\
7 & : \text { réserves } \\
8 & : \text { couvain operculé } \\
10 & : \text { rien }\end{aligned}$ \\
\hline
\end{tabular}

TABIEAU 2

(Expériences faites sur une seule ruche : ruche 5)

\begin{tabular}{|c|c|c|c|c|c|}
\hline & \multicolumn{2}{|c|}{ Nombre de larves en élevage } & \multirow{2}{*}{$\begin{array}{l}\text { Larves élevées } \\
\text { jusqu'à oper- } \\
\text { culation }\end{array}$} & \multirow{2}{*}{$\begin{array}{l}\text { Pourcentage } \\
\text { d'acceptation }\end{array}$} & \multirow{2}{*}{ État des autres cadres } \\
\hline & $\begin{array}{l}\text { Zone où se } \\
\text { trouve la reine }\end{array}$ & $\begin{array}{l}\text { Zone } \\
\text { orpheline }\end{array}$ & & & \\
\hline $\begin{array}{c}\text { Du } 26 \text { au } \\
31 / 7 / 61\end{array}$ & $\begin{array}{c}2080 \text { sur } \\
\text { cadre } 1 \\
1460 \text { sur } \\
\text { cadre } 2\end{array}$ & $\begin{array}{l}350 \text { sur } \\
\text { cadre } \gamma \\
560 \text { sur } \\
\text { cadre } 9\end{array}$ & $\begin{array}{l}350 \text { sur } \\
\text { cadre } 7 \\
560 \text { sur } \\
\text { cadre } 9\end{array}$ & $\begin{array}{r}100 \\
19\end{array}$ & $\begin{aligned} 4 & \text { : couvain } \\
& \text { operculé } \\
5 & \text { un peu } \\
& \text { de cou- } \\
& \text { vain } \\
& \text { operculé } \\
6+7+8: & \text { couvain } \\
10 \quad \text { operculé } & \text { rien }\end{aligned}$ \\
\hline $\begin{array}{c}\text { Du } 317 \text { au } \\
3 / 8 / 61\end{array}$ & $\begin{array}{l}1880 \text { sur } \\
\text { cadre } 2\end{array}$ & $\begin{array}{l}2480 \text { sur } \\
\text { cadre } 10\end{array}$ & $\begin{array}{l}130 \text { sur } \\
\text { cadre } 10\end{array}$ & 5 & $\begin{aligned} 4 & : \text { couvain } \\
& \text { operculé } \\
5 & \text { rien } \\
6+7 & : \text { couvain } \\
& \text { operculé } \\
8 & \text { rien } \\
9 & : \text { un peu } \\
& \text { de cou. } \\
& \text { vain } \\
& \text { operculé }\end{aligned}$ \\
\hline
\end{tabular}


TABLEAU 2 (Suite)

\begin{tabular}{|c|c|c|c|c|c|}
\hline & \multicolumn{2}{|c|}{ Nombre de larves en élevage } & \multirow{2}{*}{$\begin{array}{l}\text { Larves élevées } \\
\text { jusqu'à oper- } \\
\text { culation }\end{array}$} & \multirow{2}{*}{$\begin{array}{l}\text { Pourcentage } \\
\text { d'acceptation }\end{array}$} & \multirow[b]{2}{*}{ État des autres cadres } \\
\hline & $\begin{array}{c}\text { Zone où se } \\
\text { trouve la reine }\end{array}$ & $\begin{array}{l}\text { Zone } \\
\text { orpheline }\end{array}$ & & & \\
\hline $\begin{array}{c}\text { Du } 3 \text { au } \\
7 / 8 / 61\end{array}$ & $\begin{array}{l}2050 \text { sur } \\
\text { cadre } 2\end{array}$ & $\begin{array}{l}1980 \text { sur } \\
\text { cadre } 9\end{array}$ & $\begin{array}{l}520 \text { sur } \\
\text { cadre } 9\end{array}$ & 25 & $\begin{aligned} 4 & : \text { couvain } \\
& \text { operculé } \\
5 & : \text { rien } \\
6+7+8 & : \text { couvain } \\
& \text { operculé } \\
10 & : \text { rien }\end{aligned}$ \\
\hline $\begin{array}{l}\text { Du } 7 \text { au } \\
14 / 8 / 61\end{array}$ & $\begin{array}{l}1140 \text { sur } \\
\text { cadre } 2\end{array}$ & $\begin{array}{l}2580 \text { sur } \\
\text { cadre } 5 \\
1520 \text { sur } \\
\text { cadre } 10\end{array}$ & $\begin{array}{l}1200 \text { sur } \\
\text { cadre } 5 \\
1300 \text { sur } \\
\text { cadre } 10\end{array}$ & 47 & $\begin{aligned} 4 & : \text { couvain } \\
& \text { operculé } \\
7+8+9 & \text { un peu } \\
& \text { de cou- } \\
& \text { vain } \\
& \text { operculé }\end{aligned}$ \\
\hline $\begin{array}{c}\text { Du } 11 \text { au } \\
17 / 8 / 61\end{array}$ & $\begin{array}{l}500 \text { sur } \\
\text { cadre } 3\end{array}$ & $\begin{array}{l}2420 \text { sur } \\
\text { cadre } 5\end{array}$ & $\begin{array}{l}1200 \text { sur } \\
\text { cadre } 5\end{array}$ & & $\begin{aligned} 4 & : \text { réserves } \\
5 & : \text { couvain } \\
& \text { opercule } \\
6+7 & : \text { rien } \\
8+9: & \text { très peu } \\
& \text { de cou- } \\
& \text { vain } \\
& \text { opercule }\end{aligned}$ \\
\hline $\begin{array}{c}\text { Du } 18 \text { au } \\
22 / 6 / 61\end{array}$ & $\begin{array}{l}1000 \text { sur } \\
\text { cadre } 1\end{array}$ & $\begin{array}{l}3460 \text { sur } \\
\text { cadre } 7 \\
1280 \text { sur } \\
\text { cadre } 9\end{array}$ & $\begin{array}{l}600 \text { sur } \\
\text { cadre } 7 \\
1280 \text { sur } \\
\text { cadre } 9\end{array}$ & 17 & $\begin{aligned} 4 & : \text { réserves } \\
5: \text { couvain } & \text { operculé } \\
\text { oper } & \end{aligned}$ \\
\hline $\begin{array}{c}\text { Du } 22 \text { au } \\
29 / 8 / 61\end{array}$ & $\begin{array}{l}1780 \text { sur } \\
\text { cadre } 3\end{array}$ & $\begin{array}{l}1200 \text { sur } \\
\text { cadre } 7 \\
1275 \text { sur } \\
\text { cadre } 9\end{array}$ & $\begin{array}{l}540 \text { sur } \\
\text { cadre } 7 \\
100 \text { sur } \\
\text { cadre } 9\end{array}$ & 7 & $\begin{aligned} & 4: \text { réserves } \\
& \text { couvain } \\
& \text { operculé } \\
& 6: \text { couvain } \\
& \text { operculé } \\
& 8+10: \text { rien }\end{aligned}$ \\
\hline
\end{tabular}

a) Tout d'abord, remarquons qu'il est très difficile de se rendre compte du pourcentage de larves élevées sur les cadres de la zone où se trouve la reine, ceci à cause de la ponte continuelle de cette dernière et des éclosions successives qui interviennent.

Néanmoins, le cadre 2 étant continuellement remplacé pour permettre à la reine de pondre sur un cadre quasi vide, une observation journalière de l'état du couvain sur les cadres I et 3 permet de constater que pratiquement tous les oufs donnent des larves et que la transformation larves-nymphes s'effectue selon un pourcentage de l'ordre de $90 \mathrm{p}$. Ioo à $95 \mathrm{p}$. Ioo. 


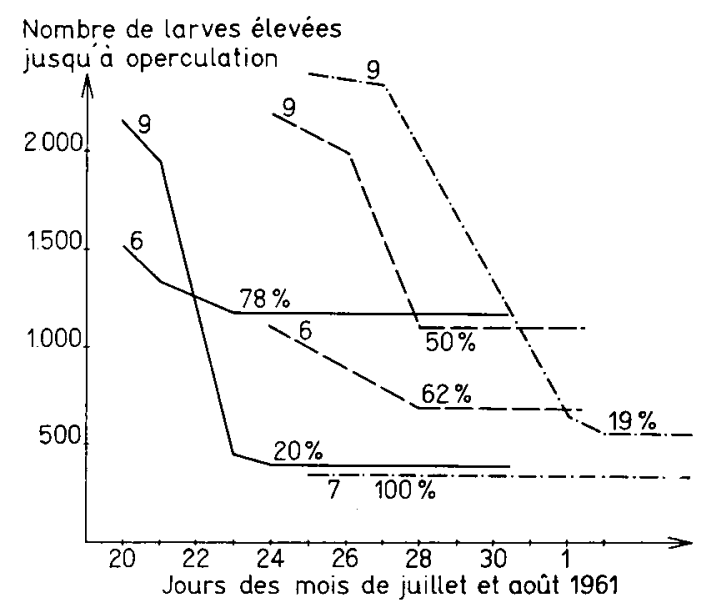

Fig. 5. - Proportion de larres élezées dans 3 ruches différentes en fonction :

${ }^{\circ}$ da nombre de laries nourries dans la zone anec la reine. - $2^{\circ}$ de leur distance à la grille à reine. - $3^{\mathbf{e}}$ du contenu du cadre (ruche - - - - )

-- - : Ruche ayant à cilever i 450 larves dans sa partie orpheline. Pourcentage d'acceptation des autres larves (en $6: 62 \%$... en $9: 50 \%$ ).

Ruche ayant à élever 2280 larves dans sa partie orpheline. Pourcentage d'acceptation des autres larves : (en $6: 78 \%$ - en $9: 20 \%)$.

Ruche ayant à ćlever 3540 larves dans sa partie proheline. - Pourcentage d'acceptation des autres larves : (en $;: 100 \%$ (presence de couvain operculé). -- en $9: 19 \%$ ).

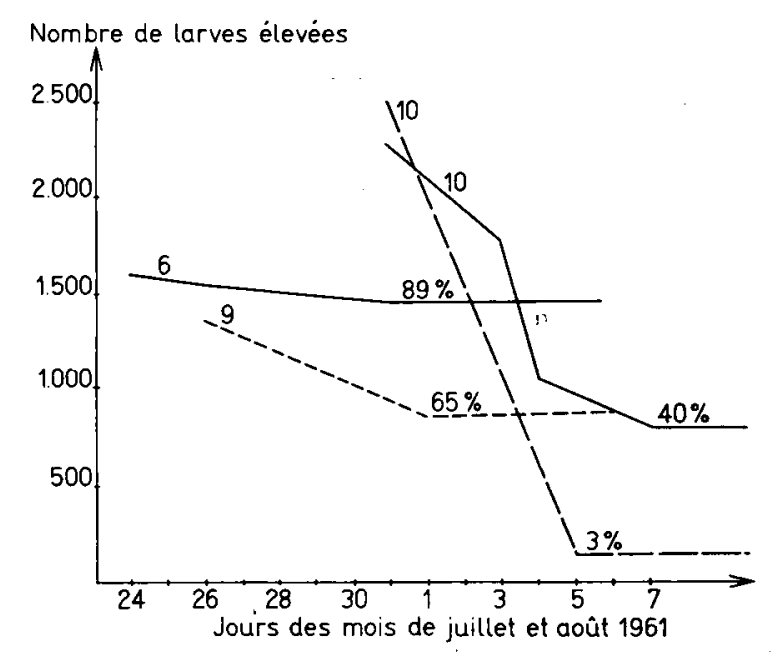

Fig. 6. - P'roportion de larves élevées dans 3 ruches différentes en fonction:

$1^{\circ}$ du nombre de larves nowries dans la sone avec la reine. $-2^{\circ}$ de leur distance a la grille à reine,

-..- : Ruche ayant à élever 660 larves dans sa partie orpheline. - Pourcentage d'acceptation en 9: $65 \%$.

: Ruche ayant à élever 2080 larves dans sa partie orpheline. - Pourcentage d'acceptation en 6 : $89 \%$.

Ruche avant à élever 2050 larres dans sa partié orpheline. Pourcentage d'acceptation en 10 : $40 \%$.

: Ruche ayant à élever 2970 larves dans sa partie orpheline. Pourcentage d'acceptation en io: $3 \%$ 
b) Il est remarquable que, quelle que soit la quantité de larves données en élevage à la ruche, il y a une nette décroissance du nombre de larves acceptées et nourries de la grille au fond de la ruche (fig. 5 et 6 ).

Ces résultats confirment donc bien notre hypothèse selon laquelle la reine constituerait véritablement un centre d'attraction pour la colonie. Elle cristalliserait l'activité des ouvrières et en particulier des nourrices, ce qui amènerait ces dernières à élever, par priorité, les larves se trouvant près de la reine.

c) L'état des rayons étant sensiblement le même dans nos expériences, il est intéressant de noter que chaque ruche semble posséder sa propre capacité d'élevage. Cela est probablement dû au nombre et à la qualité des nourrices présentes dans chaque colonie.

Toutefois, les abeilles ne semblent pas pouvoir régulariser l'élevage des larves en augmentant, par exemple, le nombre ou l'activité des nourrices lorsque le nombre des larves à nourrir est trop important. C'est ainsi que, nourrissant tout d'abord le couvain larvaire dans la zone où se trouve la reine, les abeilles ne semblent adopter les larves de la partie orpheline qu'en fonction du nombre des larves de la première zone.

Si l'on se réfère, par exemple, aux tableaux I et 2 établis pour des ruches ayant des populations sensiblement égales, on note que :

- (Tableau I), le nombre des larves présentes dans la zone possédant une reine est nettement plus grand pour la ruche 4 que pour la ruche 2 ( 2280 contre I 450 ). Cela est également vrai pour les cadres 6 de ces 2 ruches. Par suite, on note une chute brutale de l'élevage sur le cadre 9 de la ruche 4 , les abeilles élevant d'abord les larves des cadres I, 3 et 6 .

Par contre, dans la ruche 3 , le nombre des larves nourries est nettement plus faible, ce qui explique en partie le bon pourcentage d'acceptation sur le cadre 9

- (Tableau 2), dans la ruche 5 , où l'élevage a été régulièrement suivi, l'acceptation est décroissante de la grille à reine au fond de la ruche, en fonction du nombre de larves nourries sur les cadres $I, 2$ et 3 (fig. 7 ).

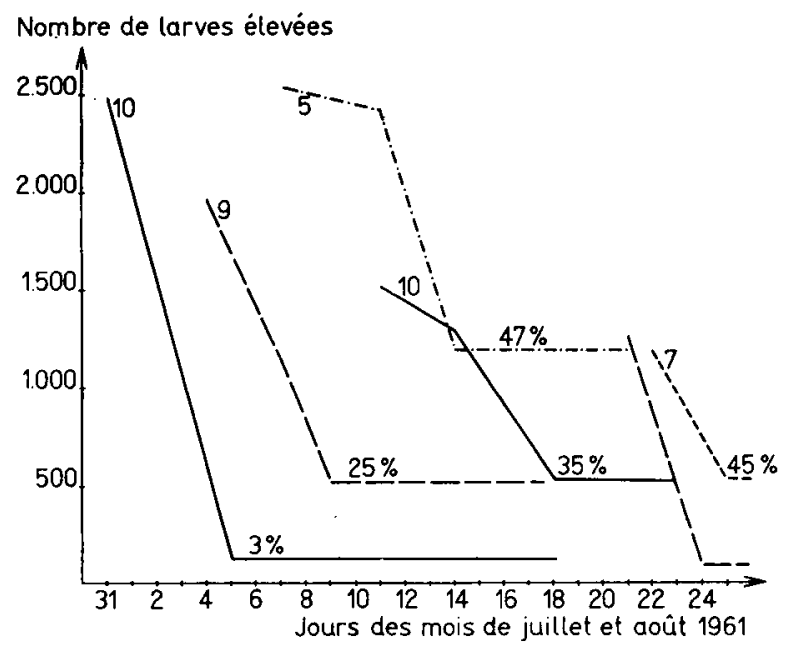

Iíl. 7. - Évolution de l'élevage des larves dans la partie orpheline t'n fonction: - $1^{\circ}$ du nombre de celles qui sont nourries dans la zone avec la reine (c). le tableau 2) - 20 de leur disiance à la reine et arx larves (cf. le tablean 2). 


\section{$2^{\circ}$ Infuence du contenu prééxistant du cadre}

Il suffit de comparer les courbes d'acceptation des larves établies d'une part pour les ruches en état de disette et, d'autre part pour les ruches que nous avons qualifiées de "saines".

Alors que dans les premières il n'y avait que peu de réserves et de couvain operculé, nous avons fait en sorte que les " ruches saines " gardent toujours une quantité de couvain operculé et de réserves qui soit sensiblement constante, ainsi que le montrent nos tableaux.

Il apparait alors clairement que la présence combinée de ces deux éléments favorise l'élevage des larves.

Nous avons alors dégagé plus nettement le rôle du couvain operculé. Pour cela, nous mettions en 2 des cadres contenant une certaine fraction de couvain operculé. Puis, lorsque la reine y avait pondu, nous l'introduisions dans la partie orpheline, à différents endroits, pour juger de l'acceptation des larves.

Nous avons alors enregistré les résultats contenus dans le tableau 3.

TABLEAU 3

\begin{tabular}{l|c|c|c}
\hline \hline & $\begin{array}{c}\text { Eufs ou larves mis } \\
\text { en élevage }\end{array}$ & Larves adoptées & $\begin{array}{c}\text { Pourcentage } \\
\text { d'acceptation }\end{array}$ \\
\cline { 2 - 4 } & 970 & 970 & 100 \\
$\begin{array}{l}\text { Cadre introduit en } 5 \\
\text { (du 20 au 26/7/61) }\end{array}$ & 350 & 350 & 100 \\
$\begin{array}{l}\text { Cadre introduit en } 7 \\
\text { (du 26/7 au 2/8/61) }\end{array}$ & 630 & 600 & 82 \\
$\begin{array}{l}\text { Cadre introduit en 7 } \\
\text { (du 21/7 au 28/8/61) } \\
\text { Cadre introduit en 9 } \\
\text { (du 3/8 au 10/8/61) }\end{array}$ & 850 & 600 & 70 \\
\hline
\end{tabular}

La présence de couvain operculé sur un cadre facilite donc l'adoption définitive des oufs et des larves qui y sont contenus.

\section{D. - VÉRIFICATION DU RÔLE PRÉDOMINANT DE IA DISTANCE REINE-LARVES}

Nous avons utilisé pour cette vérification une ruche double pouvant contenir I6 cadres. Nous avons alors établi, au milieu, une séparation ne permettant pas le passage des abeilles. Puis, nous avons versé une colonie d'abeilles de $2,5 \mathrm{~kg}$ environ dans chaque conmpartiment et nous avons isolé chaque reine sur 3 cadres aux extrémités de cette grande ruche derrière une grille. Trois jours plus tard, en prenant soin d'asperger les abeilles au sirop de miel, afin d'éviter un pillage réciproque, nous avons enlevé la cloison médiane. Nous avons ainsi constitué une grande colonie d'abeilles possédant une reine isolée à chaque extrémité et au sein de laquelle nous avons distingué arbitraitement une zone 1 et une zone 2 , chacune d'entre elles comprenant 8 cadres (fig. 8 ). 
Nous avons pu ainsi vérifier que :

- La distance reine-larves est bien un facteur essentiel dans l'acceptation et le nourrissement de ces dernières. Le pourcentage des larves élevées sur les cadres 7 et 8 est en effet nettement inférieur à celui qui se rapporte aux larves figurant sur les cadres 4,5 ou 6 (fig. 9 et Io).

- Signalons ensuite un phénomène curieux qui devra être vérifié :

De temps en temps, dès que le cadre 2 de la zone I contenait un nombre d'œufs assez important, nous l'introduisions dans la partie orpheline de la zone 2 , et inversement.

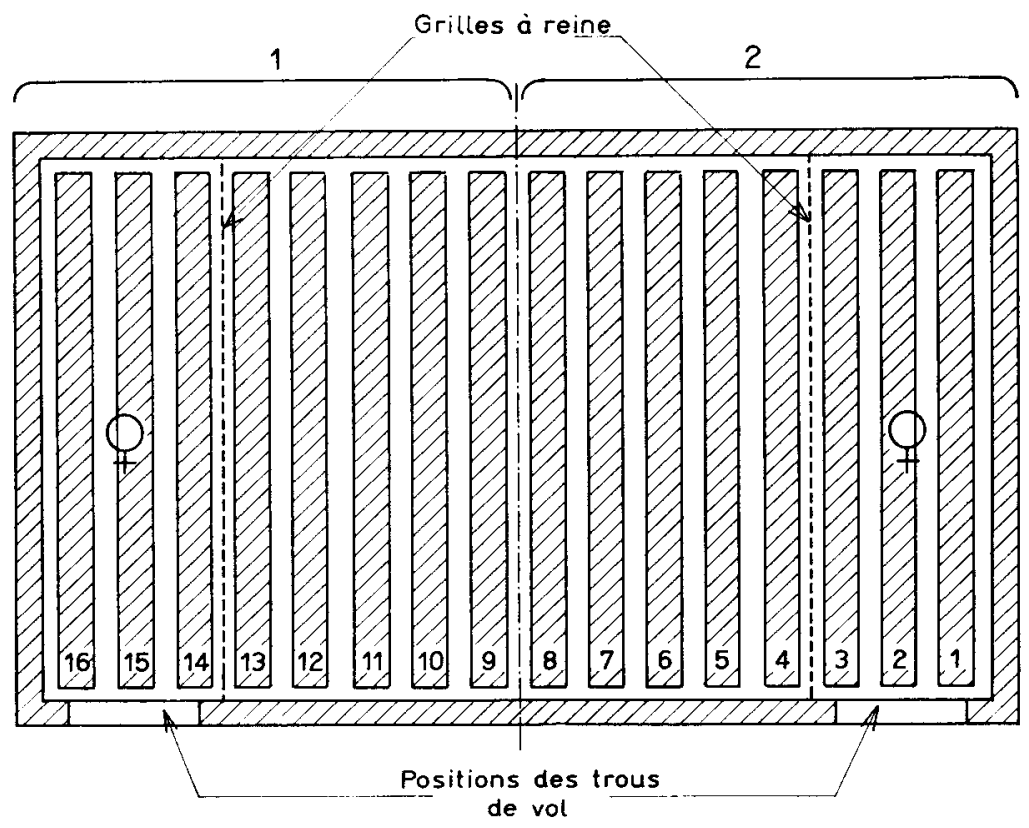

FIg. 8. - Schéma de la répartition des cadres de la ruche double

De plus, nous introduisions également dans chaque zone des cadres d'œufs provenant de ruches étrangères.

Il semblerait alors que, soumises à l'influence d'une reine, les abeilles acceptent difficilement des larves provenant d'une reine étrangère (fig. 9 et Io).

Cette constatation, si elle se trouve vérifiée, offrira un certain intérêt pratique pour les apiculteurs. En effet, on sait que pour produire la gelée royale, ces derniers prélèvent dans une même ruche des larves servant au "greffage » dans de nombreuses colonies ; ces larves sont donc élevées par des abeilles étrangères. Il est alors possible, que, si nos observations se vérifient, le faible pourcentage d'acceptation des cellules royales que l'on constate à certaines périodes de l'année, soit dû aut greffage de larves étrangères à la colonie éleveuse. Aussi conviendra-t-il de faire une étude systématique de "greffages 》 faits d'une part avec les larves d'une certaine ruche et réintroduites dans cette même ruche, et d'autre part avec des larves qu'on introduirait dans des ruches différentes. 


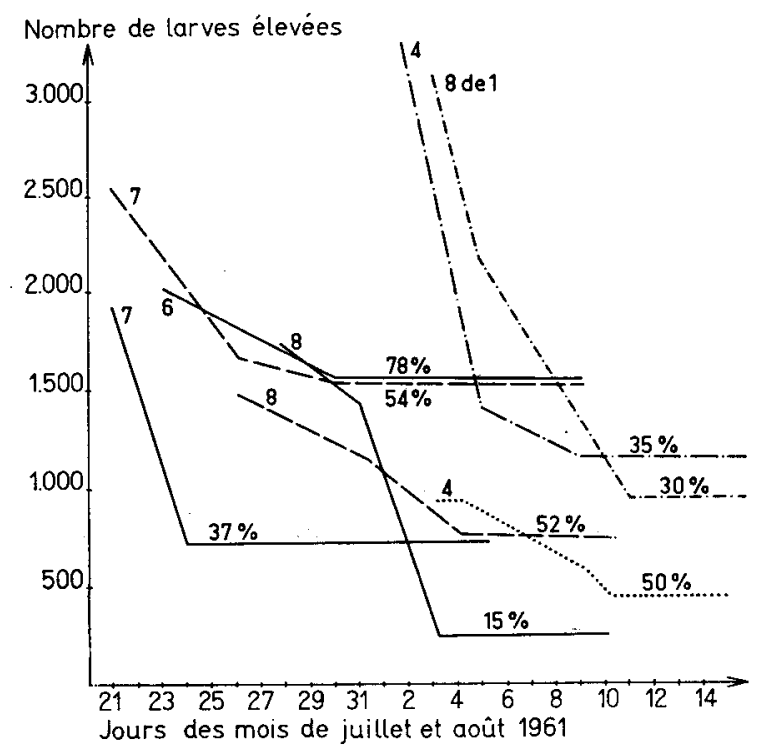

FiG. 9. - Variation de l'élevage des larves en fonction. $\mathrm{I}^{\mathrm{O}}$ de leur distance à la grille à reine dans la ruche double. $2^{\circ}$ de leur originte

Zone : Cadre passé de la zone avec reine dans la partie orpheline correspondante. Zone 1, - Cadre passé de la zone avec reine dans la partie orpheline de la zone 2 .

Zone 2 : Cadre passé de la zone avec reine dans la partie orpheline correspondante. Zone $2, \ldots \ldots$. Cadre passé de la zone avec reine dans la partie orpheline de la zone $\mathrm{I}$. ........... : Cadre venant d'une autre ruche.

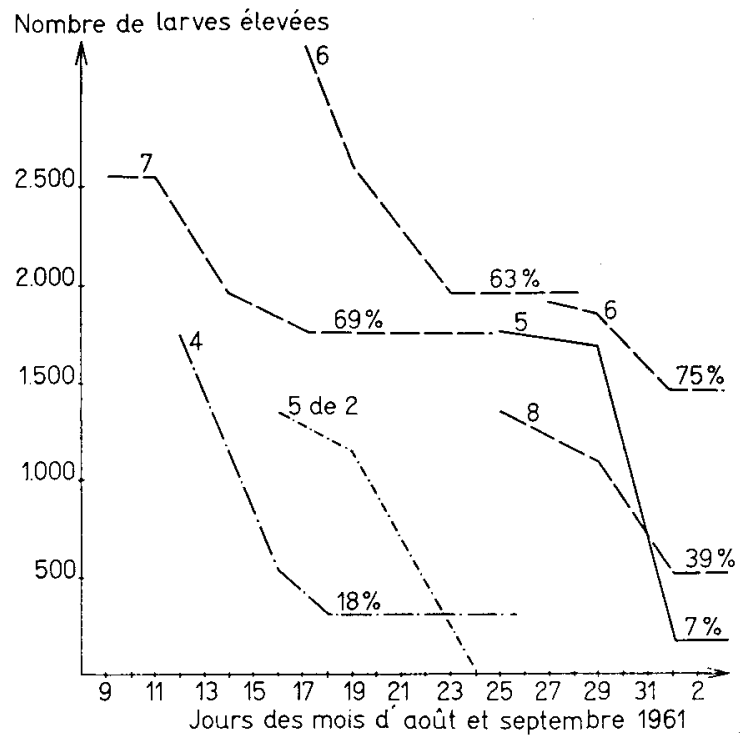

FIG. Io. - Suite de la figure 9 


\section{CONCLUSION}

Il apparaît donc que dans les conditions de nos expétiences, des œufs et des larves sont continuellement détruits ou mangés dans la ruche. (Ces conditions d'expétience sont celles que réalisent les apiculteurs qui, produisant de la gelée royale, isolent la reine derrière une grille, à l'extrémité d'une ruche.)

On pourrait penser, comme certains, qu'il apparaît des oufs non viables (HACHINOHE, JimbU, r958), ce qui est au moins possible. Mais ici, il apparaît d'une façon très nette, même en tenant compte du rendement de transformation parfois peu important, œufs-larves et larves-nymphes, défini par CHAuvin (1956), que la proportion des larves arrivant à operculation, dépend de la distance du cadre à la grille à reine Il est donc probable que le pourcentage d'acceptation oufs-larves dépend de l'activité des ouvrières, celles-ci acceptant plus facilement les larves qui se trouvent le plus près de la grille. Dès lors, la décroissance du pourcentage de larves acceptées de la grille au fond de la ruche traduit également un gradient d'activité des abeilles, confirmant notre hypothèse émise au sujet de la répartition de la nourriture dans la ruche : le centre d'activité est déplacé du côté où se trouve la reine (MONTAGNER, I962). Ilapparaît donc qu'en présence de la reine isolée à une extrémité de la ruche, les abeilles n'élèvent les larves que dans la mesure où elles ne sont pas trop éloignées de cette reine. Or, sur les cadres 7 et 8 , même 9 parfois, il y a un pourcentage de larves acceptées non négligeable. Il y aurait donc une double pulsion qui agirait sur les ouvrières et serait responsable de ce "gradient d'activité ": 1'une organique en quelque sorte et provoquée par la présence des larves (les œufs représentant un stimulus nettement plus faible) et l'autre véritablement "vitale " provoquée par la présence de la reine, qui serait nettement dominante. La reine serait donc bien le seul moteur dont dépendent toutes les activités sociales et notamment l'élevage des larves.

Dès lors, il sera intéressant de voir si la quantité d'ecto-hormone présentée par la reine élargit sa zone d'influence et permet ainsi un élevage plus important des larves.

Il apparaît enfin qu'en dehors de l'attraction de la reine, chaque ruche possède sa propre capacité d'élevage et que la présence de couvain operculé facilite 1'adoption des cufs et des larves sur le même cadre.

Reçu pour publication en juin 1962.

\section{SUMMARY}

\section{PRELIMINARY EXPERIMENTS FOR MEASURING THE BREEDING CAPACITY OF A HIVE}

The writer tries to define the chief factors influencing egg acceptance and larva rearing in bee colonies.

A study was made of the causes of inadequate breeding, firstly in food-deficient hives and then under conditions of normal nutrition and food reserves.

After having observed that each hive had its own specific breeding capacity and that the bees did not seem to have the power to change this to any significant degree, the following two factors were found to have determinant effects :

I) the presence of an operculated brood comb ;

2) the distance of the larvae from the queen.

There was evidence that a gradient of activity existed to which the bees conformed very closely under the conditions of the experiments. 


\section{RÉFÉRENCES BIBL,IOGRAPHIQUES}

Chauvin R., 1948. Biologie de l'Abeille. Revue générale jusqu'en i956. Ann. Abeille, 1, 4I-67.

Chauvin R., ig6o. Progrès récents dans la biologie de l'Abeille. Ann. Abeille, 1, 6-I4.

Hachinoe Y., JIMBU M., I958. Occurrence of non viable eggs in the honey-bees. Bull. Nat. Inst. agric. Sci. Ser., 6, 14, 123-130.

Montagner H., I962. Sur les transports de réserves et les transports de couvain dans la ruche. Ins. Soc., t. IX, 2, I2I-I28.

Musalewsky B. M., 1935. Bruteifer und Schwarmgrösse. Arch. Bienenk., 16, 181-7•

Taranov G. F., I946. Zoologisk. Zhurnal., 25, 25 I-262. 